Draft Version July 17, 2018

Preprint typeset using $\mathrm{LAT}_{\mathrm{E}} \mathrm{X}$ style emulateapj v. 5/2/11

\title{
XO-2B: A HOT JUPITER WITH A VARIABLE HOST STAR THAT POTENTIALLY AFFECTS ITS MEASURED TRANSIT DEPTH
}

\author{
RoBert T. ZELLEM \\ Department of Planetary Sciences, Lunar and Planetary Laboratory, University of Arizona, 1629 East University Boulevard, University \\ of Arizona, Tucson, AZ, 85721, USA; rzellem@lpl.arizona.edu
}

\author{
Caitlin A. Griffith \\ Department of Planetary Sciences, Lunar and Planetary Laboratory, University of Arizona, 1629 East University Boulevard, University
} of Arizona, Tucson, AZ, 85721, USA; griffith@lpl.arizona.edu

KyLE A. PEARson

Department of Astronomy, Steward Observatory, University of Arizona, 933 North Cherry Avenue, Tucson, AZ, 85721, Currently Department of Physics and Astronomy, Northern Arizona University, Flagstaff, AZ, 86001, USA

JAKE D. TURNER

Department of Planetary Sciences, University of Arizona, 933 North Cherry Avenue, Tucson, AZ, 85721, USA, Currently Department of Astronomy, University of Virginia, Charlottesville, VA, 22904, USA

Gregory W. HEnRY

Center of Excellence in Information Systems, Tennessee State University, 3500 John A. Merritt Blvd., PO Box 9501, Nashville, TN 37209, USA

Michael H. Williamson

Center of Excellence in Information Systems, Tennessee State University, 3500 John A. Merritt Blvd., PO Box 9501, Nashville, TN 37209 , USA

\section{RYLEIGH FITZPATRICK}

Department of Astronomy, Steward Observatory, University of Arizona, 933 North Cherry Avenue, Tucson, AZ, 85721, USA

JOHANNA K. TESKE

Department of Astronomy, Steward Observatory, University of Arizona, 933 North Cherry Avenue, Tucson, AZ, 85721, USA, Currently Carnegie DTM, 5241 Broad Branch Road, NW, Washington, DC 20015, USA, Carnegie Origins Fellow, jointly appointed by Carnegie DTM \& Carnegie Observatories

\section{LAUREN I. BIDDLE}

Department of Astronomy, Steward Observatory, University of Arizona, 933 North Cherry Avenue, Tucson, AZ, 85721, USA, Currently Lowell Observatory, 1400 West Mars Hill Road, Flagstaff, AZ 86001, USA

Draft version July 1\%, 2018

\section{ABSTRACT}

The transiting hot Jupiter XO-2b is an ideal target for multi-object photometry and spectroscopy as it has a relatively bright $(V$-mag $=11.25) \mathrm{K} 0 \mathrm{~V}$ host star $(\mathrm{XO}-2 \mathrm{~N})$ and a large planet-to-star contrast ratio $\left(\mathrm{R}_{p} / \mathrm{R}_{s} \approx 0.015\right)$. It also has a nearby (31.21") binary stellar companion (XO-2S) of nearly the same brightness $(V-m a g=11.20)$ and spectral type $(\mathrm{G} 9 \mathrm{~V})$, allowing for the characterization and removal of shared systematic errors (e.g., airmass brightness variations). We have therefore conducted a multiyear (2012-2015) study of XO-2b with the University of Arizona's 61" (1.55 m) Kuiper Telescope and Mont4k CCD in the Bessel U and Harris B photometric passbands to measure its Rayleigh scattering slope to place upper limits on the pressure-dependent radius at, e.g., 10 bar. Such measurements are needed to constrain its derived molecular abundances from primary transit observations. We have also been monitoring XO-2N since the 2013-2014 winter season with Tennessee State University's Celestron-14 (0.36 m) automated imaging telescope to investigate stellar variability, which could affect XO-2b's transit depth. Our observations indicate that XO-2N is variable, potentially due to cool star spots, with a peak-to-peak amplitude of $0.0049 \pm 0.0007 \mathrm{R}$-mag and a period of $29.89 \pm 0.16$ days for the $2013-2014$ observing season and a peak-to-peak amplitude of $0.0035 \pm 0.0007 \mathrm{R}$ mag and $27.34 \pm 0.21$ day period for the 2014-2015 observing season. Because of the likely influence of XO-2N's variability on the derivation of XO-2b's transit depth, we cannot bin multiple nights of data to decrease our uncertainties, preventing us from constraining its gas abundances. This study 
demonstrates that long-term monitoring programs of exoplanet host stars are crucial for understanding host star variability.

Subject headings: planets and satellites: individual (XO-2b) - methods:analytical — atmospheres — radiative transfer - planets and satellites: general

\section{INTRODUCTION}

The exoplanet $\mathrm{XO}-2 \mathrm{~b}$ is arguably typical of the more extensively measured transiting exoplanets, considering its basic characteristics and available observations. It is a Jupiter-sized body that orbits a K0V star at a distance of $0.0369 \pm 0.002 \mathrm{AU}$ and a period of 2.6 days (Burke et al. 2007). The relatively bright ( $V$ mag $=11.25 ;$ Benavides et al. 2010) host star, XO-2N, has a southern binary companion, $\mathrm{XO}-2 \mathrm{~S}$, that resides too far away (4600 AU) to affect the planetary orbit (Burke et al. 2007). Both stars have relatively high metallicities and C/O ratios (Teske et al. 2013), as well as the same stellar type and brightness. Thus, XO-2S provides an ideal photometric reference star for transit observations of the exoplanet and host star.

Ground-based observations of XO-2b at optical wavelengths reveal the presence of both sodium and potassium (Sing et al. 2011a, 2012). Broad-band photometry measures the planetary radius at which its atmosphere becomes optically thick in the Sloan $z$ band (Fernandez et al. 2009) and in the broad 0.4-0.7 $\mu \mathrm{m}$ band (Burke et al. 2007). A large, multi-platform, ground-based study of the XO-2 system finds evidence for variability in both $\mathrm{XO}-2 \mathrm{~N}$ and $\mathrm{XO}-2 \mathrm{~S}$ and records fourteen R-band photometry primary transits of XO-2b (Damasso et al. 2015). Secondary eclipse Spitzer/IRAC observations suggest that XO-2b has a weak thermal inversion (Machalek et al. 2009). The Hubble Space Telescope (HST) with NICMOS obtained a XO-2b transmission spectrum at near-IR wavelengths of $1.2-1.8 \mu \mathrm{m}$, potentially indicating the presence of water vapor in its atmosphere (Crouzet et al. 2012).

Molecular abundances are difficult to determine from near-IR primary transit measurements because the derived mixing ratios depend on the radius assumed as a function of pressure level (Tinetti et al. 2010; Benneke \& Seager 2012, 2013). Griffith (2014) finds that the extinction coefficient depends exponentially on the pressure-dependent radius, indicating that high precision measurements of exoplanetary radii are essential for the interpretation of transit spectra. Note that the derived mixing ratios are not sensitive, separately, to uncertainties in the host star's radius, because primary transits measure the ratio of the planet-to-star radius (Griffith 2014). Thus the derived planet's radius scales to the assumed stellar radius, which we take to be $0.964 R_{\text {Sun }}$ for the host star XO-2N. Gas abundances determined from primary transit data are also sensitive to the temperature profile of the planet, but to a much lesser extent (Griffith 2014).

Here we present repeated ground-based primary transit measurements of XO-2b's atmospheric transmission with the University of Arizona's 61" (1.55 m) Kuiper Telescope in the Harris B $(330-550 \mathrm{~nm})$ and Bessell U (303-417 nm) photometric bands, where the spectrum is devoid of molecular features. If the exoplanet is cloudless, the opacities within these bands are established by
$\mathrm{H}_{2}$ Rayleigh scattering, and thus the atmospheric density structure and mean molecular weight, rather than atomic and molecular features. The mean molecular weight is already well known through XO-2b's density (Narita et al. 2011; Burke et al. 2007), which indicates a $\mathrm{H}_{2}$-He based atmosphere. Constraints on XO-2b's thermal profile are provided by previous Spitzer/IRAC observations (Machalek et al. 2009). If clouds are present, an upper limit to the radius is obtained, as further discussed below.

Our study also includes a nightly monitoring program of the host star XO-2N for variability with Tennessee State University's C14 (0.36 m) Automatic Photoelectric Telescope (AIT) at Fairborn Observatory (Henrv 1999). These R-band photometric measurements suggest that $\mathrm{XO}-2 \mathrm{~N}$ is indeed variable, likely the result of star spot activity, and could influence XO-2b's transit depth (e.g. Pont et al. 2008; Agol et al. 2010; Carter et al. 2011; Désert et al. 2011; Sing et al. 2011a; McCullough et al. 2014; Oshagh et al. 2014; Damasso et al. 2015). However, it is unclear how much this variability alters XO2b's signal as all our Kuiper/Mont4k measurements agree to $\sim 1 \sigma$. Regardless, XO-2N's variability prevents us from binning the multiple years of Kuiper/Mont4k data in order to achieve a higher precision measurement of XO-2b's planet-to-star radius ratio. As a result, we cannot strongly constrain its molecular abundances.

\section{OBSERVATIONS AND DATA REDUCTION}

Photometric measurements were conducted at the University of Arizona's 61" (1.55 m) Kuiper Telescope, equipped with the Mont4k CCD, a 4096×4096 pixel sensor with a $9.7^{\prime} \times 9.7^{\prime}$ field of view. Bessel U-band measurements alone were obtained on 2012 January 5, 2013 January 21, and 2013 February 24 (UT). Harris B-band measurements alone were obtained on 2012 October 29 and 2014 January 30 (UT). Simultaneous U and B-band measurements were taken on 2012 December 10, 2014 February 12, 2015 January 18, and 2015 February 8 (UT). Typical seeing was $\sim 1.5 "$. At the telescope, we binned the pixels by $3 \times 3$ to shorten the readout time and achieve a plate scale of 0.43 " / pixel. To ensure accurate time-keeping, an on-board clock was automatically synchronized with GPS every few seconds throughout the observational period.

To help characterize the out-of-transit baseline, each set of observations began $\sim 1-2$ hours prior to transit ingress and ended $\sim 1-2$ hours after egress. The flux from XO-2N was measured simultaneously with the flux from 8 additional stars on the CCD. Each image is biassubtracted and flat-fielded with 10 flats and bias frames, using standard IRAF reduction procedures (Tody 1993). Although 8 reference stars were imaged, only the binary companion, $\mathrm{XO}-2 \mathrm{~S}$, is used as a comparison star to map out systematic errors (e.g., airmass), because it is nearby (31.21") and of similar stellar type (G9V; Benavides et al. 2010) and brightness $(V-\mathrm{mag}=11.20$; Høg et al. 2000) to the host star. In addition, we distin- 
guish a short periodicity in one of the stars within the field of view (RA: 7:48:05.44; Dec: $+50: 15: 56.1$ ), thereby ruling it out as a reference star.

To extract the time-varying flux of the target and comparison stars, we use the Exoplanet Data Reduction Pipeline (ExoDRPL, Pearson et al. 2014). Aperture radius sizes ranging from 7 to 14 pixels (0.98" to $1.96 "$ ") with steps of 0.1 pixel (0.014") are explored to incorporate sufficient flux from each star and ensure that no contaminating light from nearby stars is present in the radial profile. A constant sky annulus with an inner and outer radius of 16 and 20 pixels (2.24" and 2.8 "), respectively, both larger than the target aperture, prevent the inclusion of any background star light. The aperture radii that produce the lowest scatter in the raw $\mathrm{XO}-2 \mathrm{~N}$ light curves were used to create the final light curves. This selection process minimizes both the point-to-point scatter as well as airmass effects. For each night, the light curves of $\mathrm{XO}-2 \mathrm{~N}$ are then divided by the light curves of $\mathrm{XO}-2 \mathrm{~S}$ to remove shared systematic errors, the largest of which is airmass.

The light curves are derived from the data with the analytic equations of Mandel \& Agol (2002) to generate a model transit. A Levenberg-Marquardt (LM) non-linear least squares minimization (Press et al. 1992) via the Interactive Data Language mpfit function (Markwardt 2009) provides an initial local fit of the model light curves to our data. During the entire analysis, the time of mid-transit $\left(T_{c}\right)$, planet-to-star radius ratio $\mathrm{R}_{p} / \mathrm{R}_{s}$, and out-of-transit normalized flux $a$ are left as the only free parameters. The orbital period, inclination, scaled semi-major axis, eccentricity, and quadratic limb darkening coefficients remained fixed for this analysis and are listed in Table 1. In cases where the reduced chi-squared $\left(\chi_{r}^{2}\right)$ of the data to the initial-fit model is greater than unity, we multiply the photometric error bars by $\sqrt{\chi_{r}^{2}}$ to compensate for the underestimated observational errors (Bruntt et al. 2006; Southworth et al. 2007) in order to produce a global best-fit solution with a reduced $\chi^{2}$ equal to unity. This inflation technique captures some of the additional non-photon (red) noise in the data; the time-correlated component of the red noise is captured later in the analysis with a prayer bead.

To find a global fit solution, we employ 4 simultaneous Markov Chain Monte Carlo (MCMC; Ford 2005) chains of 200,000 links (steps) seeded by randomly perturbing the best-fit parameters from the LM analysis. Each MCMC chain draws from a prior Gaussian distribution determined by the initial LM fit and tuned so that each fitted parameter has an acceptance ratio of $\sim 0.25$ to maximize chain efficiency (Ford 2005). After running each MCMC chain, we define the burn-in point for each chain as where the $\chi^{2}$ value first falls below the median of all the $\chi^{2}$ values in the chain, and remove all links prior to this point. A Gelman-Rubin test (Gelman \& Rubin 1992) finds a scale reduction factor $\leq 1.001$ for all fitted parameters (Ford 2005), suggesting that all chains converge to the same global solutions.

Since the MCMC draws from a Gaussian prior distribution and assumes that every measurement is uncorrelated, it can underestimate the uncertainties in the derived parameters, particularly if there is non-Gaussian red noise (Carter \& Winn 2009). Therefore we use a residual permutation, or "prayer bead", method (e.g., Jenkins et al. 2002; Southworth 2008; Bean et al. 2008; Winn et al. 2008) to give a more robust estimate of the parameter uncertainties. The prayer bead method circularly permutes the residuals around the best-fit MCMC solution, and solves for the transit depth, mid-transit time, and out-of-transit normalized flux $a$ with a LM for each permutation. In this way $n$ (where $n$ is the number of data points) new "simulated" lightcurves are formed in order to generate new posterior distributions of the parameters. To remain conservative, we choose the larger of the two uncertainties from either the MCMC or the prayer bead as the final error bars for each derived parameter and allow our uncertainties to be non-symmetric (e.g., Todorov et al. 2012). The final model fits to the data are presented in Table 2 and Figures $1,4$.

\section{EFFECTS OF STELLAR VARIABILITY}

Initial analyses of the $2012 \mathrm{U}$ and B-band data indicated 1- $\sigma$ agreement between the derived $R_{p} / R_{s}$ values. This consistency seemed to reinforce previous studies that concluded that XO-2 is a non-active system. For example, Burke et al. (2007) found that all of their Extended Team XO-2b light curves agreed to $\sim 0.5 \%$. In addition, Sing et al. (2011a) found no evidence for occulted starspots in their GTC/OSIRIS spectrophotometric study of $\mathrm{XO}-2 \mathrm{~b}$. However we found that our out-oftransit baseline (as indicated by the out-of-transit normalized flux $a$; see Table 2) varied $0.6 \%$ in the $U$ and $2.4 \%$ in the B, suggesting that the host star $\mathrm{XO}-2 \mathrm{~N}$ is variable. For comparison, Burke et al. (2007) found that $\mathrm{XO}-2 \mathrm{~N}$ was $93.76 \%(0.07 \pm 0.008 \mathrm{mag})$ the brightness of $\mathrm{XO}-2 \mathrm{~S}$ in the B-band, whereas we find that $\mathrm{XO}-2 \mathrm{~N}$ varies between $92.258 \%$ and $93.839 \%$ of the brightness of $\mathrm{XO}-2 \mathrm{~S}$.

While we do not see any conclusive evidence for starspot crossings in any of our light curves, unocculted starspots can cause a transit depth to deepen, resulting in the retrieval of an incorrect $\mathrm{R}_{p} / \mathrm{R}_{s}$ measurement and an incorrect interpretation of the exoplanet's atmosphere (e.g., Pont et al. 2008; Agol et al. 2010; Carter et al. 2011; Désert et al. 2011; Sing et al. 2011b). To investigate the possibility of photometric variability in $\mathrm{XO}$ $2 \mathrm{~N}$ and $\mathrm{XO}-2 \mathrm{~S}$, we made nightly observations of both components throughout the 2013-2014 and 2014-2015 observing seasons with the Tennessee State University Celestron-14 (C14) Automated Imaging Telescope (AIT) at Fairborn Observatory in southern Arizona (e.g., Henry 1999; Eaton et al. 2003). We acquired $\sim 170$ and $\sim 100$ nightly photometric measurements in the Cousins Rband during the two observing seasons. We compute differential magnitudes of $\mathrm{XO}-2 \mathrm{~N}$ and $\mathrm{XO}-2 \mathrm{~S}$ with respect to the mean brightness of several of the most stable comparison stars in the CCD field of view and then perform separate frequency analyses of XO-2N and XO$2 \mathrm{~S}$ for each observing season (Table 3 and Figs. 5-8). A few outliers were removed in each analysis, based on 3$\sigma$ filtering. Further details of our C14 data acquisition and reduction procedures can be found in the paper by Sing et al. (2015), which describes a similar analysis of the exoplanet-host star WASP-31.

The 2013-2014 observations of the northern component, XO-2N, have more scatter than the 2014-2015 season (Table 3 and Figs. 5 \& 6). The frequency 
Table 1

Fixed Model Values

\begin{tabular}{|c|c|c|}
\hline Parameter & Values & Reference \\
\hline \multicolumn{3}{|l|}{ XO-2b's Orbital Parameters } \\
\hline Period (days) & 2.61586178 & Sing et al. (2011a) \\
\hline Inclination $\left({ }^{\circ}\right)$ & 88.01 & Crouzet et al. (2012) \\
\hline $\mathrm{a} / \mathrm{R}_{s}$ & 7.986 & Crouzet et al. (2012) \\
\hline Eccentricity & 0 & Crouzet et al. (2012) \\
\hline \multicolumn{3}{|l|}{ Host Star XO-2N Parameters } \\
\hline $\mathrm{T}_{e f f}$ & $5343 \mathrm{~K}$ & Teske et al. (2013) \\
\hline $\log g(\operatorname{cgs})$ & 4.49 & Teske et al. (2013) \\
\hline$[\mathrm{Fe} / \mathrm{H}]$ & 0.39 & Teske et al. (2013) \\
\hline Limb darkening coefficients (U-band) & $0.98884840,-0.13270563$ & Eastman et al. (2013) \\
\hline Limb darkening coefficients (B-band) & $0.81461289,0.026193820$ & Eastman et al. (2013) \\
\hline
\end{tabular}

Please note that the Teske et al. (2013) values used here agree with a more contemporary study by Damasso et al. (2015) to $1 \sigma$.

Table 2

Derived Parameters

\begin{tabular}{|c|c|c|c|c|c|c|c|c|c|c|}
\hline \multirow{2}{*}{$\begin{array}{c}\begin{array}{c}\text { Date } \\
(\mathrm{UT})\end{array} \\
\text { Bessel U-b }\end{array}$} & \multirow{2}{*}{$\mathbf{R}_{p} / \mathbf{R}_{s}$} & \multicolumn{2}{|c|}{ Uncertainty } & \multirow{2}{*}{$\begin{array}{l}\text { Mid-transit time } \\
\left(T_{c}-2456000 \mathrm{JD}\right)\end{array}$} & \multicolumn{2}{|c|}{ Uncertainty } & \multirow[t]{2}{*}{$a^{\dagger}$} & \multicolumn{2}{|c|}{ Uncertainty } & \multirow{3}{*}{$\begin{array}{c}\sigma / \sigma_{\text {photon }} \\
3.71\end{array}$} \\
\hline & & & & & & & & & & \\
\hline 2012 Jan 5 & 0.1037 & -0.0035 & +0.0041 & -68.2337 & -0.0030 & +0.0016 & 0.88703 & -0.00032 & +0.00041 & \\
\hline 2012 Dec 10 & 0.1047 & -0.0024 & +0.0028 & 271.8283 & -0.0025 & +0.0014 & 0.88254 & -0.00024 & +0.00024 & 3.17 \\
\hline 2013 Jan 21 & 0.1035 & -0.0019 & +0.0023 & 313.6810 & -0.0018 & +0.0024 & 0.93235 & -0.00014 & +0.00031 & 3.84 \\
\hline 2013 Feb 24 & 0.1059 & -0.0015 & +0.0015 & 347.6865 & -0.0006 & +0.0006 & 0.87921 & -0.00023 & +0.00023 & 3.90 \\
\hline 2014 Feb 12 & 0.1072 & -0.0020 & +0.0020 & 700.8276 & -0.0012 & +0.0017 & 0.87379 & -0.00029 & +0.00028 & 3.36 \\
\hline 2015 Jan 18 & 0.1031 & -0.0035 & +0.0045 & 1040.8883 & -0.0022 & +0.0039 & 0.87210 & -0.00019 & +0.00033 & 2.79 \\
\hline 2015 Feb 8 & 0.1087 & -0.0051 & +0.0033 & 1061.8169 & -0.0040 & +0.0023 & 0.86897 & -0.00021 & +0.00034 & 2.98 \\
\hline \multicolumn{11}{|c|}{ Harris B-band } \\
\hline 2012 Oct 29 & 0.1054 & -0.0015 & +0.0016 & 229.9736 & -0.0005 & +0.0010 & 0.93839 & -0.00014 & +0.00027 & 3.64 \\
\hline 2012 Dec 10 & 0.1030 & -0.0021 & +0.0018 & 271.8288 & -0.0023 & +0.0008 & 0.92409 & -0.00018 & +0.00019 & 3.83 \\
\hline 2014 Jan 30 & 0.1042 & -0.0033 & +0.0075 & 687.7504 & -0.0017 & +0.0031 & 0.93024 & -0.00050 & +0.00057 & 6.08 \\
\hline 2014 Feb 12 & 0.1076 & -0.0038 & +0.0039 & 700.8291 & -0.0030 & +0.0028 & 0.92258 & -0.00039 & +0.00044 & 5.20 \\
\hline 2015 Jan 18 & 0.1038 & -0.0041 & +0.0023 & 1040.8889 & -0.0012 & +0.0034 & 0.93782 & -0.00022 & +0.00019 & 6.19 \\
\hline 2015 Feb 8 & 0.1030 & -0.0012 & +0.0028 & 1061.8164 & -0.0007 & +0.0025 & 0.93196 & -0.00010 & +0.00019 & 3.71 \\
\hline
\end{tabular}

$\dagger$ Out-of-transit normalized flux, effectively measures the brightness ratio of the host star XO-2N to the comparison (and binary companion) star XO-2S

spectrum in the middle panel of Figure 5 reveals lowamplitude brightness variability in $\mathrm{XO}-2 \mathrm{~N}$ with a period of $29.9 \pm 0.16$ days. A least-squares sine fit to the phase curve in the bottom panel of Fig. 5 shows a peak-topeak amplitude of $0.0049 \pm 0.0007 \mathrm{mag}$, or $0.53 \%$, a $7 \sigma$ detection. We take this to be the rotation period of the northern component made visible by the rotational modulation of starspots across the face of the star. This period is consistent with the range of 29-44 days predicted from the stellar radius of $0.97 R_{\odot}$ and $v \sin i=1.4 \pm 0.3$ from the discovery paper of Burke et al. (2007). Brightness variation in the northern component is reduced in the 2014-2015 observing season, but the data essentially confirm the results from the first season. The 2.5 day difference in the two periods likely results from spot evolution on the timescale of the rotation period.

The observations of the southern component, XO-2S, have very low scatter, 0.0025 and $0.0023 \mathrm{mag}$ in the first and second seasons, respectively (Table 3 and Figs. 7 $\& 8)$. These values are consistent with our measurement precision for a single observation, as determined by inter-comparison of the most constant stars in the CCD frame. Period analyses of XO-2S reveal no sig- nificant periodicity in either observing season. Our observed levels of variability of $\mathrm{XO}-2 \mathrm{~N}$ and $\mathrm{XO}-2 \mathrm{~S}$ are consistent with their activity levels, $\log R_{H K}^{\prime}$, of $-4.91 \pm 0.01$ and $-5.02 \pm 0.01$, respectively, given by Damasso et al. (2015). However our period analyses are inconsistent with those of Damasso et al. (2015), despite their use of a similarly-sized telescope $(40 \mathrm{~cm})$ relative to our study $(36 \mathrm{~cm})$ and our two monitoring programs partially overlapping in the 2013-2014 season. This discrepancy probably arises due to the difference in sampling: Damasso et al. (2015) monitored the XO-2 system for 42 nights in the $I$-band over the $2013-2014$ season while our program measured it at a much higher cadence over 171 nights in the $2013-2014$ season and 97 nights in the 2014-2015 season.

Assuming XO-2N's variability is constant over the last 4 years of observation 1 , we can plot XO-2b's transit depth in each filter vs. XO-2N's $R$-band variability

1 Please note that since the coverage of star spots varies with time, the amplitudes of the variability observed in the 2013-2014 and 2014-2014 seasons are not necessarily applicable to all of our transit observations which were recorded in the 2011-2015 winter seasons (Table 2). 

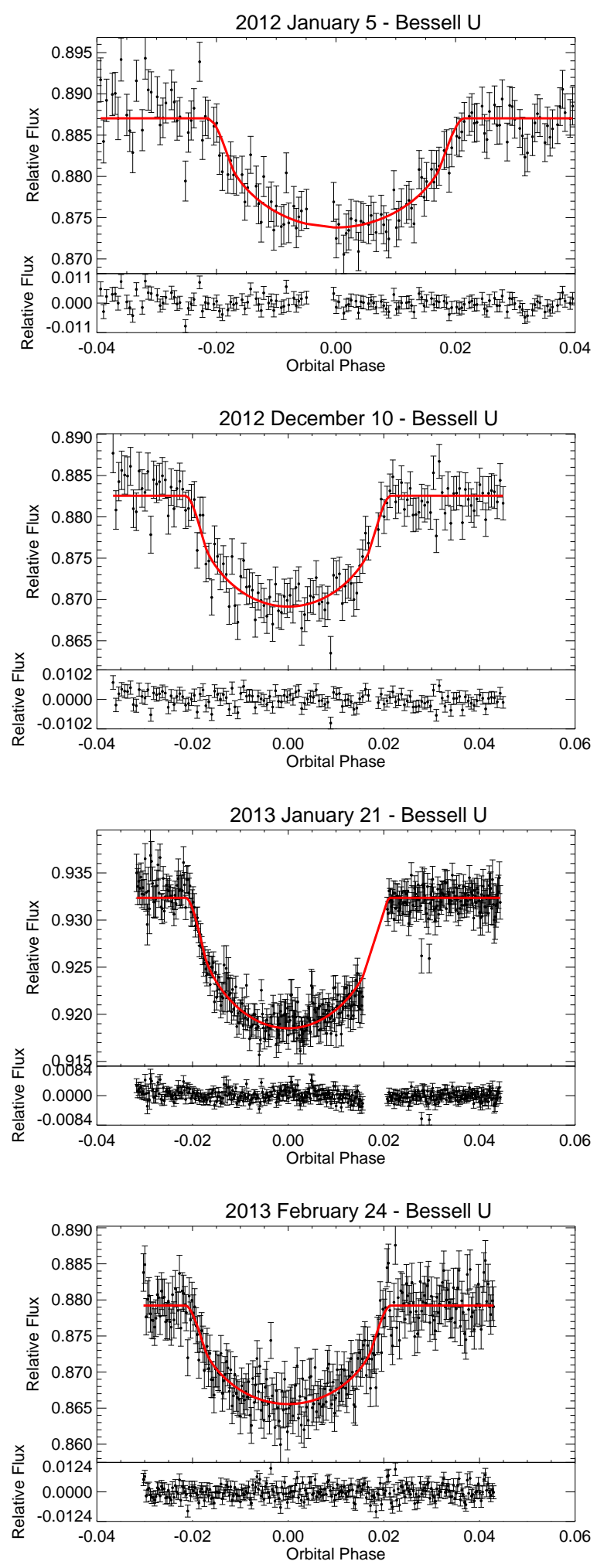

Figure 1. Bessell U-band light curves from the 2012-2013 observing seasons showing the transit of $\mathrm{XO}-2 \mathrm{~b}$ with their best-fit models indicated with the red line. The $1 \sigma$ error bars include the readout noise, the poisson noise, and the flat-fielding error. The residuals for each light curve are in the lower panel.
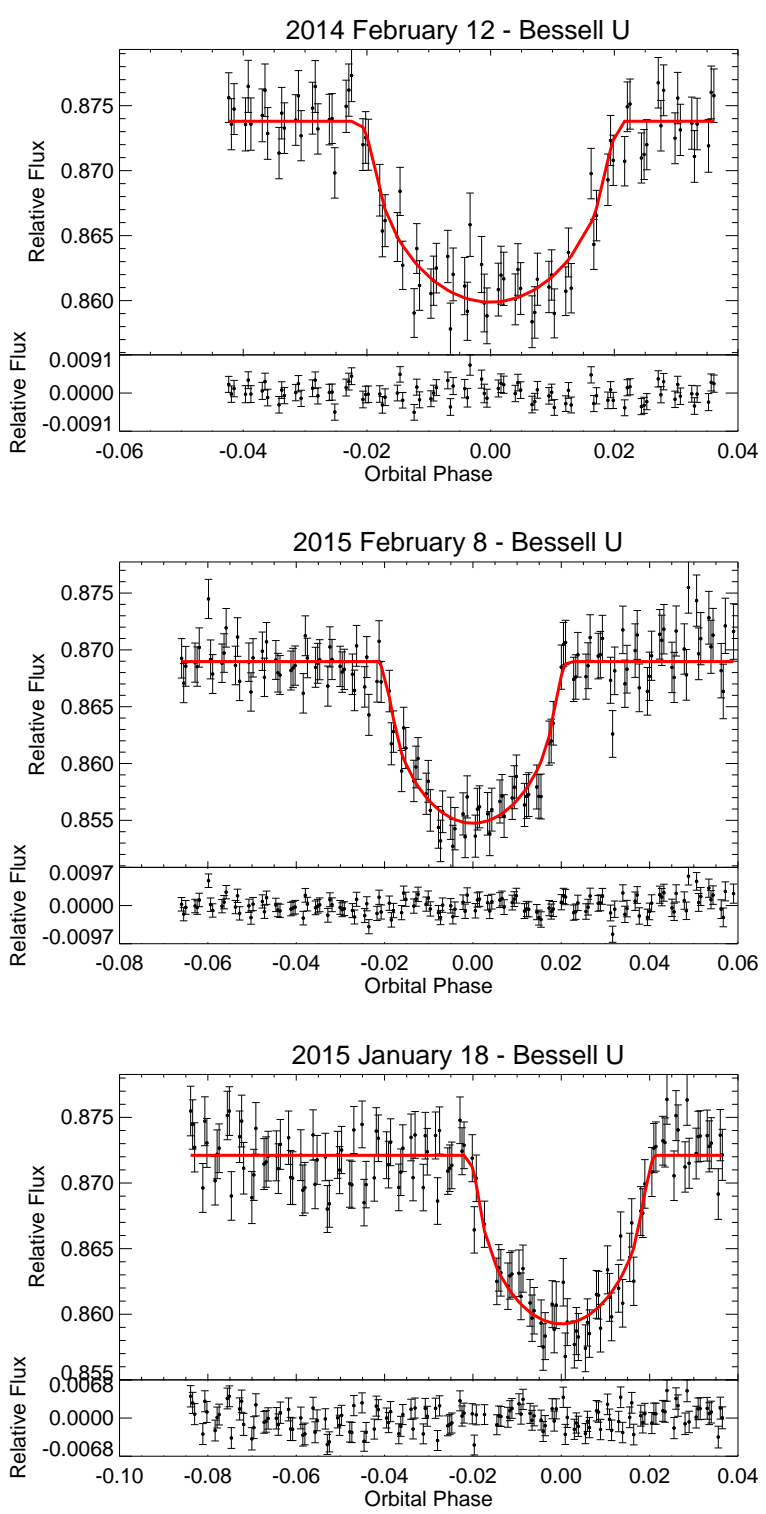

Figure 2. Bessell U-band light curves from the 2014-2015 observing seasons showing the transit of $\mathrm{XO}-2 \mathrm{~b}$ with their best-fit models indicated with the red line. The $1 \sigma$ error bars include the readout noise, the poisson noise, and the flat-fielding error. The residuals for each light curve are in the lower panel.

across the 2013-2014 and 2014-2015 seasons (Figs. 9 and 10). In the U-band, the transit depth has a -0.60 correlation with the host star's 2013-2014 variability and a 0.45 correlation with the host star's 2014-2015 variability. In the B-band, the correlation is 0.19 with the host star's 2013-2014 variability and -0.68 with the host star's 2014-2015 variability. These plots suggest a correlation between the measured transit depth and host star variability. However since all of the Kuiper/Mont4k transit depths agree to $\sim 1 \sigma$, we cannot quantify how stellar variability is affecting the derived transit depth with any statistical significance. Since the variation in the measured transit depth is potentially being influenced by the host star's activity, the multiple transit depth measurements cannot be binned together to achieve higher precision. Thus we cannot constrain XO-2b's transit depth 

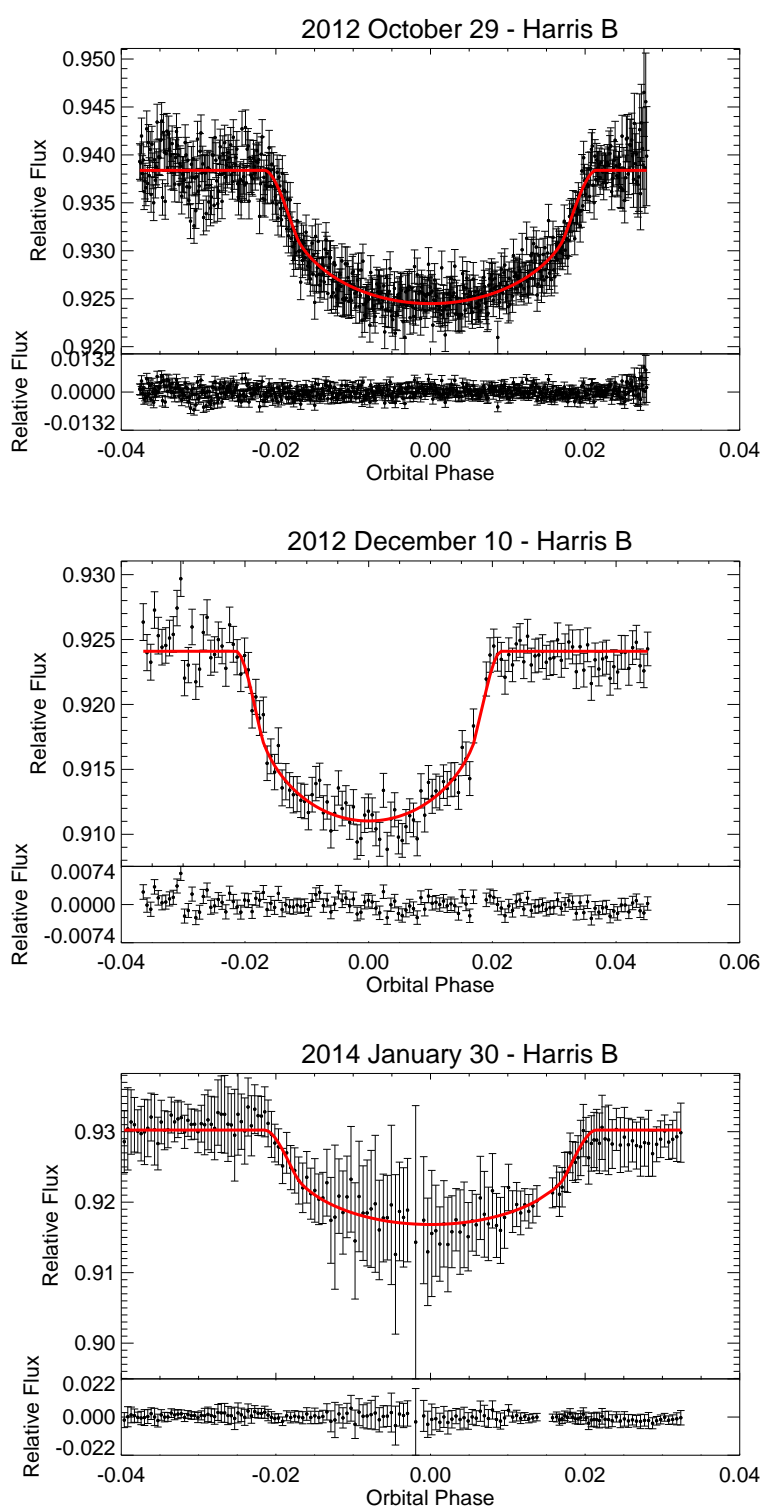

Figure 3. Harris B-band light curves from the 2012-2014 seasons showing the transit of $\mathrm{XO}-2 \mathrm{~b}$, following the same protocol as Figure 1

beyond $11.72 \%$ in the $\mathrm{U}$-band and $11.17 \%$ in the B-band.

\section{INTERPRETATION}

To assess the effects of disparate values of the measured radius, we consider several radiative transfer models of XO-2b's data. We include Rayleigh scattering, absorption due to water and pressure-induced hydrogen absorption. The atmosphere is assumed to be cloudless. Since XO-2b has an equilibrium temperature between HD 189733b and HD 209458b, we adopt a thermal profile intermediate between those of HD 189733b and HD 209458b (Moses et al. 2011). The line parameters for $\mathrm{H}_{2} \mathrm{O}$ are calculated from the parameters of Rothman et al. (2009); Tashkun et al. (2003); Barber et al. (2006), while hot pressure-induced $\mathrm{H}_{2}$ absorption derives from Borysow (2002); Zheng \& Borysow
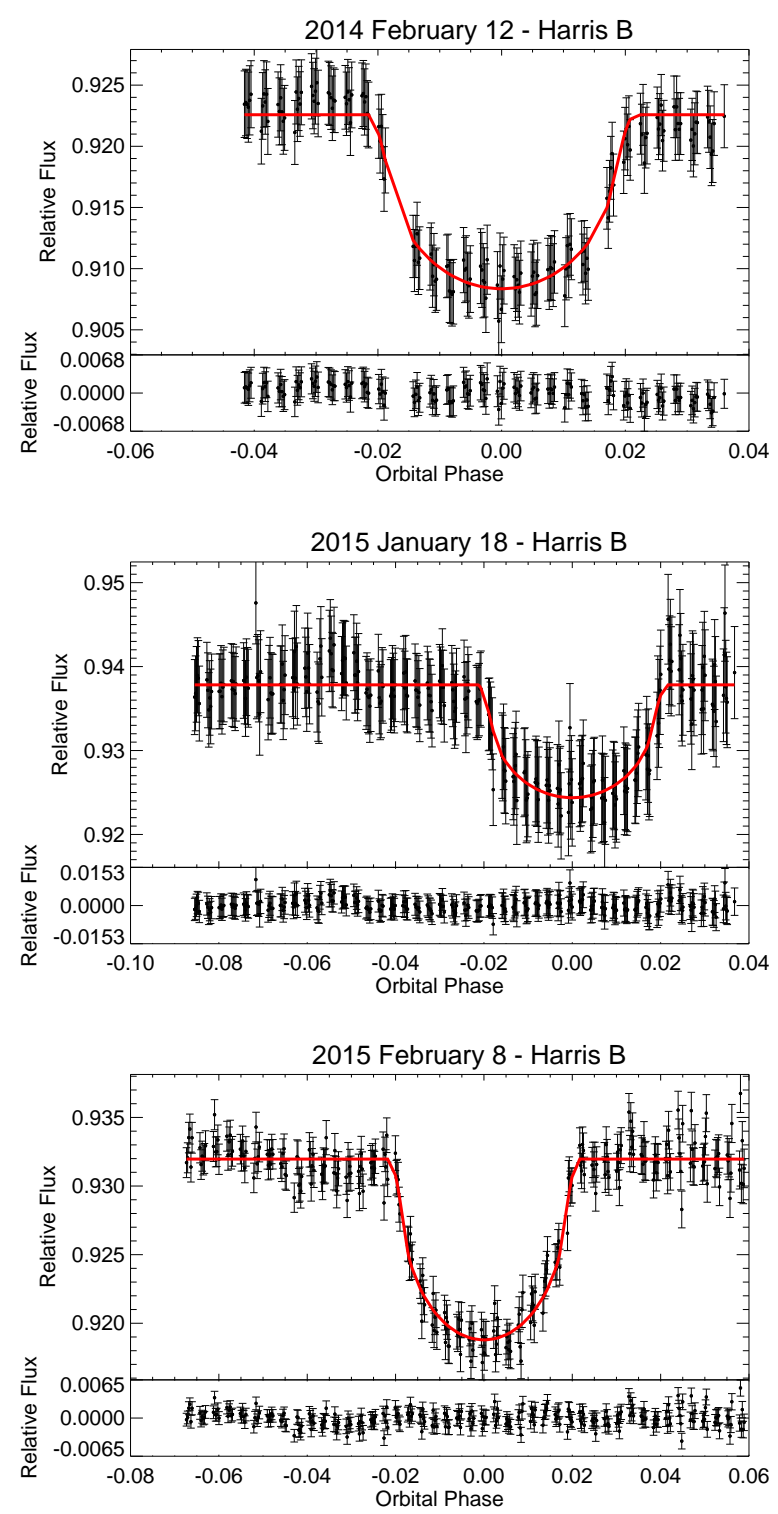

Figure 4. Harris B-band light curves from the 2014-2015 seasons showing the transit of $\mathrm{XO}-2 \mathrm{~b}$, following the same protocol as Figure 1

(1995). As shown in Figure 11, uncertainties in the XO2b's U and B-band light curve depths lead to strong uncertainties in the pressure-dependent radius, which in turn leads to poor constraints on the composition, even in the event of highly precise near-IR measurements, currently possible with Hubble/WFC3 (Griffith 2014). Our observations of $\mathrm{XO}-2 \mathrm{~b}$ indicate that its host star variability prevents us from combining data from multiple nights in order to reduce our uncertainties. These limitations indicate the great need for measurements on different platforms, monitoring programs with one instrument, and simultaneous observations at different wavelengths, which sample the same effective radius (and can then be interpreted together).

\section{CONCLUSIONS}

Our observations indicate that XO-2b's host star, $\mathrm{XO}-2 \mathrm{~N}$, is variable with a peak-to-peak amplitude of 
Table 3

SUMMARY OF AIT PHOTOMETRIC OBSERVATIONS XO-2N AND XO-2S

\begin{tabular}{ccccccc}
\hline \hline Star & $\begin{array}{c}\text { Observing } \\
\text { Season }\end{array}$ & $N_{\text {obs }}$ & $\begin{array}{c}\text { Date Range } \\
(\text { HJD }-2,400,000)\end{array}$ & $\begin{array}{c}\sigma \\
(\mathrm{mag})\end{array}$ & $\begin{array}{c}\text { Period } \\
\text { (days) }\end{array}$ & $\begin{array}{c}\text { Full Amplitude } \\
(\mathrm{mag})\end{array}$ \\
\hline $\mathrm{XO}-2 \mathrm{~N}$ & $2013-2014$ & 171 & $56572-56808$ & 0.0035 & $29.89 \pm 0.16$ & $0.0049 \pm 0.0007$ \\
$\mathrm{XO}-2 \mathrm{~N}$ & $2014-2015$ & 97 & $56945-57154$ & 0.0027 & $27.34 \pm 0.21$ & $0.0035 \pm 0.0007$ \\
$\mathrm{XO}-2 \mathrm{~S}$ & $2013-2014$ & 174 & $56572-56813$ & 0.0025 & $\ldots$ & $\ldots$ \\
$\mathrm{XO}-2 \mathrm{~S}$ & $2014-2015$ & 105 & $56945-57154$ & 0.0023 & $\ldots$ & $\ldots$
\end{tabular}
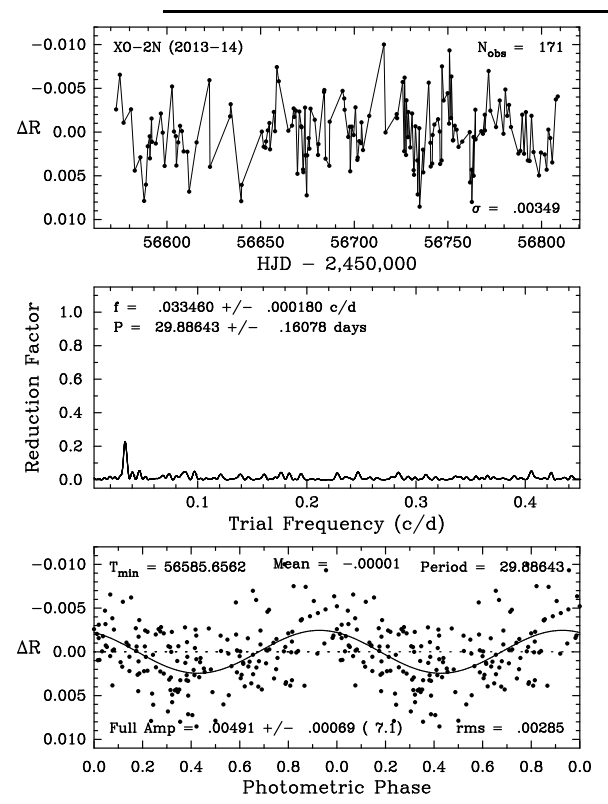

Figure 5. Top: 171 nightly Cousins $R$-band photometric observations of XO-2N from the 2013-2014 observing season, acquired with the C14 Automated Imaging Telescope at Fairborn Observatory. Middle: Frequency spectrum of the nightly observations finds a best period of $29.89 \pm 0.16$ days. Bottom: The observations phased with the best period give a full amplitude of $0.0049 \pm 0.0007$ mag, a $7 \sigma$ result.

$0.0049 \pm 0.0007 \mathrm{mag}$ in $\mathrm{R}$ and a $29.89 \pm 0.16$ day period in the 2013-2014 season and a peak-to-peak amplitude of $0.0035 \pm 0.0007 \mathrm{R}$-mag and a period of $27.34 \pm 0.21$ days for the 2014-2015 observing season. This host star variability potentially influences XO-2b's transit depths. Due to the stellar variability, our data cannot be binned to reduce uncertainties. Our study not only demonstrates that ground-based monitoring of a host star for variability is crucial for transit observations, but also that visible and near-IR observations must be taken at the same time to insure that the same stellar conditions are probed.

The work by R. T. Zellem, C. A. Griffith, K. A. Pearson, J. D. Turner, and J. K. Teske was supported by the NASA Atmospheres Program.

GWH and MHW thank Lou Boyd for his many years of APT support at Fairborn Observatory and acknowledge support from NASA, NSF, Tennessee State University, and the State of Tennessee through its Centers of Excellence program.

RTZ thanks Mario Damasso, Nikole K. Lewis, Mahmoudreza Oshagh, and Mark R. Swain for their helpful discussions.
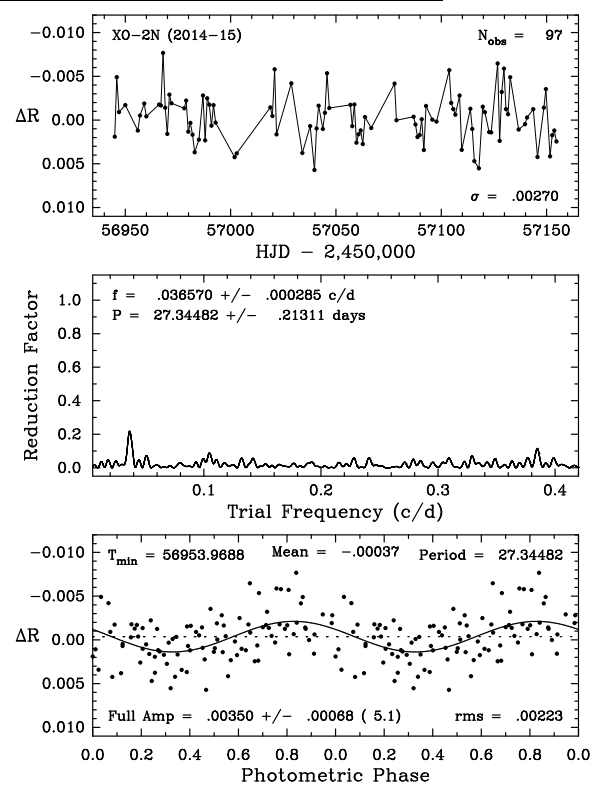

Figure 6. Top: 97 nightly Cousins $R$-band photometric observations of XO-2N from the 2014-2015 observing season. Middle: Frequency spectrum of the nightly observations finds a best period of $27.34 \pm 0.21$ days. Bottom: The observations phased with the best period give a full amplitude of $0.0035 \pm 0.0007 \mathrm{mag}$, a $5 \sigma$ result.
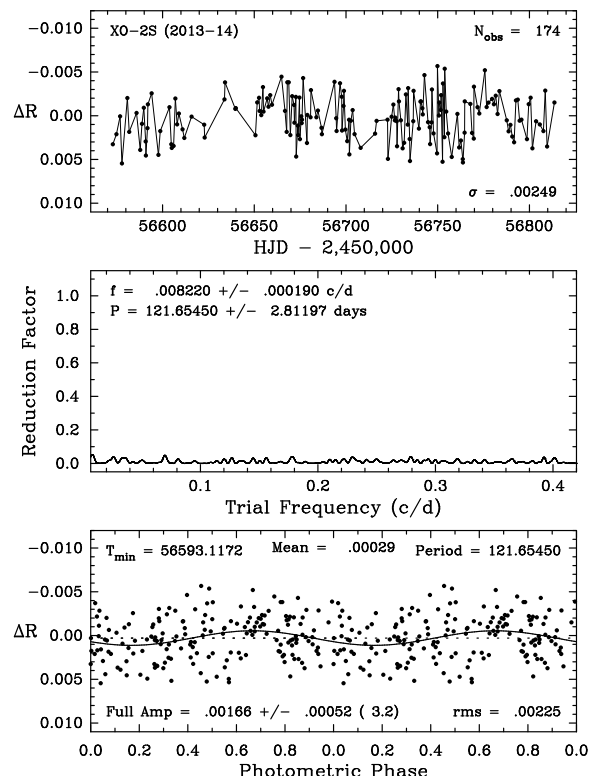

Figure 7. Top: The nightly Cousins $R$-band photometric observations of XO-2S from the 2013-2014 observing season. Middle: Frequency spectrum of these observations finds no significant periodicity between 2 and 200 days. Bottom: Phase plot shows only a spurious period for the southern component in the 2013-2014 observing season. 

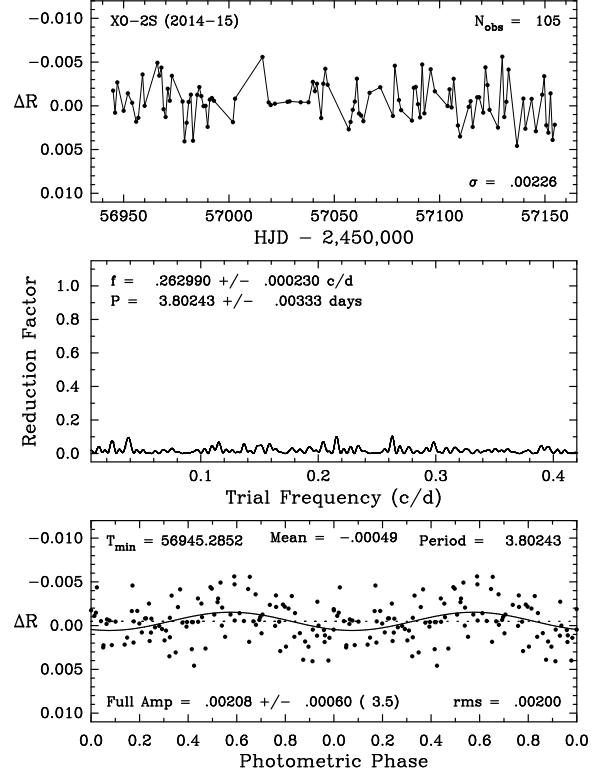

Figure 8. Top: The nightly Cousins $R$-band photometric observations of XO-2S from the 2014-2015 observing season. Middle: Frequency spectrum of the nightly observations finds no significant periodicity between 2 and 200 days. Bottom: Phase plot shows only a spurious period for the southern component in 2014-2015.

We thank the anonymous referee for their helpful comments and suggestions.

\section{REFERENCES}

Agol, E., Cowan, N. B., Knutson, H. A., Deming, D., Steffen, J. H., Henry, G. W., \& Charbonneau, D. 2010, ApJ, 721, 1861 Barber, R. J., Tennyson, J., Harris, G. J., \& Tolchenov, R. N. 2006, MNRAS, 368, 1087

Bean, J. L., Benedict, G. F., Charbonneau, D., Homeier, D., Taylor, D. C., McArthur, B., Seifahrt, A., Dreizler, S., \& Reiners, A. 2008, A\&A, 486, 1039

Benavides, R., Rica, F., Reina, E., Castellanos, J., Naves, R., Lahuerta, L., \& Lahuerta, S. 2010, Journal of Double Star Observations, 6, 30

Benneke, B., \& Seager, S. 2012, ApJ, 753, 100

-. 2013, ApJ, 778, 153

Borysow, A. 2002, A\&A, 390, 779

Bruntt, H., Southworth, J., Torres, G., Penny, A. J., Clausen, J. V., \& Buzasi, D. L. 2006, A\&A, 456, 651

Burke, C. J., McCullough, P. R., Valenti, J. A., Johns-Krull, C. M., Janes, K. A., Heasley, J. N., Summers, F. J., Stys, J. E., Bissinger, R., Fleenor, M. L., Foote, C. N., García-Melendo, E. Gary, B. L., Howell, P. J., Mallia, F., Masi, G., Taylor, B., \& Vanmunster, T. 2007, ApJ, 671, 2115

Carter, J. A., \& Winn, J. N. 2009, ApJ, 704, 51

Carter, J. A., Winn, J. N., Holman, M. J., Fabrycky, D., Berta, Z. K., Burke, C. J., \& Nutzman, P. 2011, ApJ, 730, 82

Crouzet, N., McCullough, P. R., Burke, C., \& Long, D. 2012, ApJ, 761, 7

Damasso, M., Biazzo, K., Bonomo, A. S., Desidera, S., Lanza, A. F., Nascimbeni, V., Esposito, M., Scandariato, G., Sozzetti, A., Cosentino, R., Gratton, R., Malavolta, L., Rainer, M., Gandolfi, D., Poretti, E., Zanmar Sanchez, R., Ribas, I., Santos, N., Affer, L., Andreuzzi, G., Barbieri, M., Bedin, L. R. Benatti, S., Bernagozzi, A., Bertolini, E., Bonavita, M., Borsa, F., Borsato, L., Boschin, W., Calcidese, P., Carbognani, A., Cenadelli, D., Christille, J. M., Claudi, R. U., Covino, E., Cunial, A., Giacobbe, P., Granata, V., Harutyunyan, A., Lattanzi, M. G., Leto, G., Libralato, M., Lodato, G., Lorenzi, V., Mancini, L., Martinez Fiorenzano, A. F., Marzari, F., Masiero, S., Micela, G., Molinari, E., Molinaro, M., Munari, U., Murabito, S., Pagano, I., Pedani, M., Piotto, G., Rosenberg, A., Silvotti, R., \& Southworth, J. 2015, A\&A, 575, A111
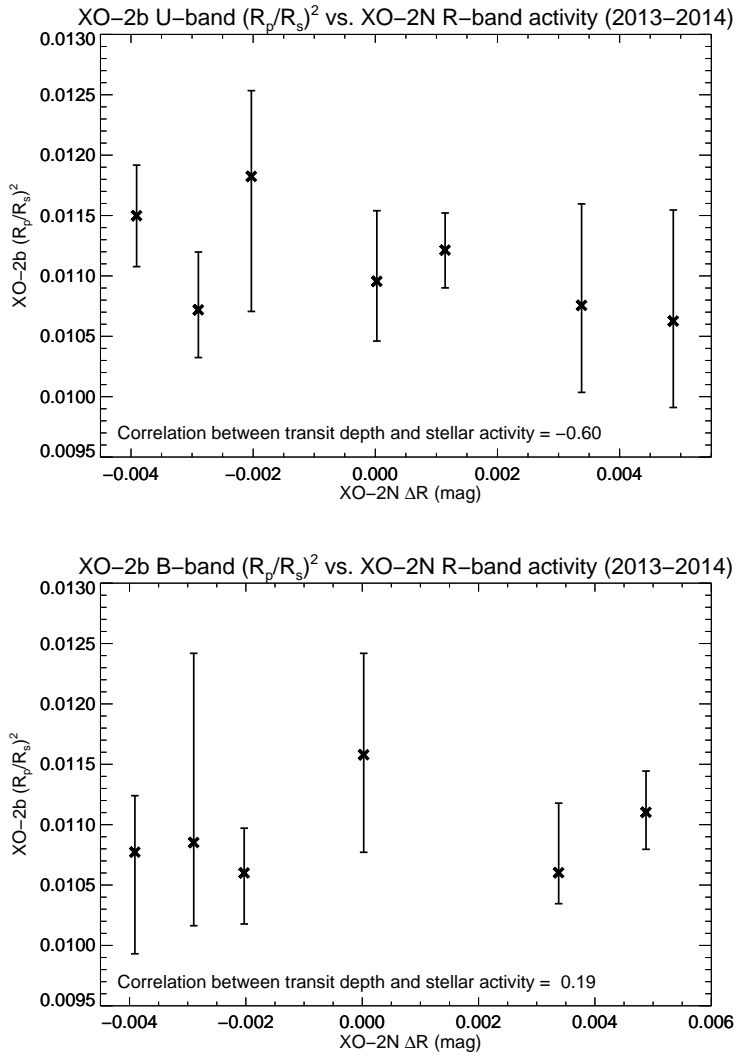

Figure 9. XO-2b's Bessel U (top) and Harris B (bottom) photometric light curve depths $\left(\mathrm{R}_{p} / \mathrm{R}_{s}\right)^{2}$ recorded by the Kuiper 61 " telescope from $2012-2015$ vs. XO-2N's $R$-band variability extrapolated from the AIT monitoring over the 2013-2014 season (Fig. 5). These plots suggest a correlation between the measured transit depth and host star variability in the U-band $(-0.60)$ but not in the B-band (0.19). Since XO-2N's variability is likely affecting the derived transit depth in both passbands, we cannot bin the multiple nights of data together to achieve higher precision.

Désert, J.-M., Sing, D., Vidal-Madjar, A., Hébrard, G., Ehrenreich, D., Lecavelier Des Etangs, A., Parmentier, V., Ferlet, R., \& Henry, G. W. 2011, A\&A, 526, A12

Eastman, J., Gaudi, B. S., \& Agol, E. 2013, PASP, 125, 83

Eaton, J. A., Henry, G. W., \& Fekel, F. C. 2003, in Astrophysics and Space Science Library, Vol. 288, Astrophysics and Space Science Library, ed. T. D. Oswalt, 189

Fernandez, J. M., Holman, M. J., Winn, J. N., Torres, G., Shporer, A., Mazeh, T., Esquerdo, G. A., \& Everett, M. E. 2009, AJ, 137, 4911

Ford, E. B. 2005, AJ, 129, 1706

Gelman, A., \& Rubin, D. B. 1992, Statistical Science, 7, pp. 457

Griffith, C. A. 2014, Phil. Trans. R. Soc. A, 372

Henry, G. W. 1999, PASP, 111, 845

Høg, E., Fabricius, C., Makarov, V. V., Urban, S., Corbin, T., Wycoff, G., Bastian, U., Schwekendiek, P., \& Wicenec, A. 2000, A\&A, 355, L27

Jenkins, J. M., Caldwell, D. A., \& Borucki, W. J. 2002, ApJ, 564, 495

Machalek, P., McCullough, P. R., Burrows, A., Burke, C. J., Hora, J. L., \& Johns-Krull, C. M. 2009, ApJ, 701, 514

Mandel, K., \& Agol, E. 2002, ApJ, 580, L171

Markwardt, C. B. 2009, in Astronomical Society of the Pacific Conference Series, Vol. 411, Astronomical Data Analysis Software and Systems XVIII, ed. D. A. Bohlender, D. Durand, \& P. Dowler, 251

McCullough, P. R., Crouzet, N., Deming, D., \& Madhusudhan, N. 2014, ApJ, 791, 55 

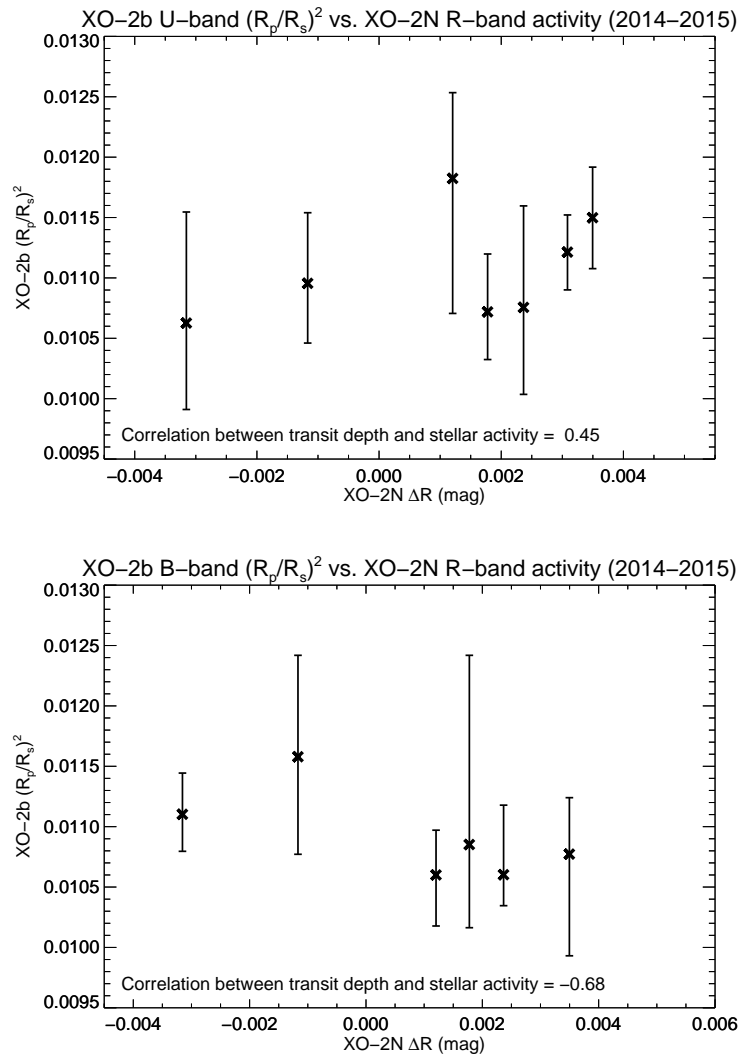

Figure 10. XO-2b's Bessel U (top) and Harris B (bottom) photometric light curve depths $\left(\mathrm{R}_{p} / \mathrm{R}_{s}\right)^{2}$ recorded by the Kuiper 61 " telescope from $2012-2015$ vs. XO-2N's $R$-band variability extrapolated from the AIT monitoring over the 2014-2015 season (Fig. 6). These plots suggest a correlation between the measured transit depth and host star variability in both the U-band (0.45) and the B-band $(-0.68)$.

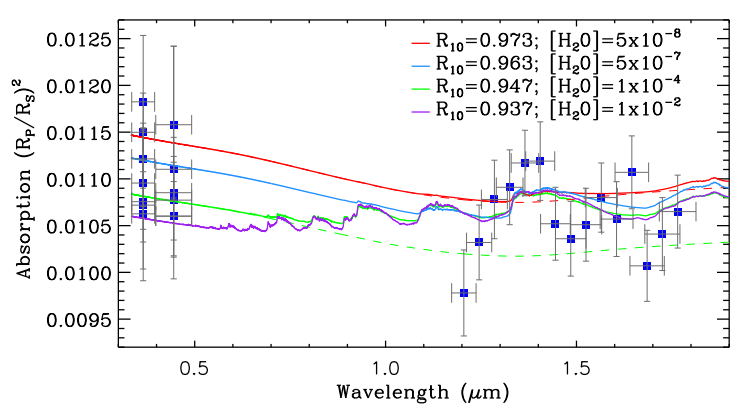

Figure 11. Hubble/NICMOS measurements (Crouzet et al. 2012) of XO-2b's primary transit spectrum $(1.2-1.8 \mu \mathrm{m})$ and visible photometry from the 61" Kuiper Telescope $(0.3-0.5 \mu \mathrm{m})$ are compared to calculated model spectra. Due to the variance in the transit depths measured both with Hubble and the 61", there is not a single radiative transfer model that can interpret all of the data simultaneously, preventing us from determining XO-2b's 10 bar radius $\left(\mathrm{R}_{10}\right)$ and placing strong constraints on its water abundance $\left[\mathrm{H}_{2} \mathrm{O}\right]$ (e.g., see Griffith 2014).

Moses, J. I., Visscher, C., Fortney, J. J., Showman, A. P., Lewis, N. K., Griffith, C. A., Klippenstein, S. J., Shabram, M., Friedson, A. J., Marley, M. S., \& Freedman, R. S. 2011, ApJ, 737,15

Narita, N., Hirano, T., Sato, B., Harakawa, H., Fukui, A., Aoki, W., \& Tamura, M. 2011, PASJ, 63, L67

Oshagh, M., Santos, N. C., Ehrenreich, D., Haghighipour, N.,

Figueira, P., Santerne, A., \& Montalto, M. 2014, A\&A, 568, A99

Pearson, K. A., Turner, J. D., \& Sagan, T. G. 2014, New Astronomy, 27, 102

Pont, F., Knutson, H., Gilliland, R. L., Moutou, C., \& Charbonneau, D. 2008, MNRAS, 385, 109

Press, W. H., Teukolsky, S. A., Vetterling, W. T., \& Flannery, B. P. 1992, Numerical Recipes in C: The Art of Scientific Computing. Second Edition

Rothman, L. S., Gordon, I. E., Barbe, A., Benner, D. C., Bernath, P. F., Birk, M., Boudon, V., Brown, L. R.

Campargue, A., Champion, J.-P., Chance, K., Coudert, L. H., Dana, V., Devi, V. M., Fally, S., Flaud, J.-M., Gamache, R. R., Goldman, A., Jacquemart, D., Kleiner, I., Lacome, N., Lafferty, W. J., Mandin, J.-Y., Massie, S. T., Mikhailenko, S. N., Miller, C. E., Moazzen-Ahmadi, N., Naumenko, O. V., Nikitin, A. V., Orphal, J., Perevalov, V. I., Perrin, A., Predoi-Cross, A., Rinsland, C. P., Rotger, M., Šimečková, M., Smith, M. A. H., Sung, K., Tashkun, S. A., Tennyson, J., Toth, R. A., Vandaele, A. C., \& Vander Auwera, J. 2009, Journal of Quantitative Spectroscopy and Radiative Transfer, 110, 533

Sing, D. K., Désert, J.-M., Fortney, J. J., Lecavelier Des Etangs, A., Ballester, G. E., Cepa, J., Ehrenreich, D., López-Morales, M., Pont, F., Shabram, M., \& Vidal-Madjar, A. 2011a, A\&A, 527, A73

Sing, D. K., Huitson, C. M., Lopez-Morales, M., Pont, F., Désert, J.-M., Ehrenreich, D., Wilson, P. A., Ballester, G. E., Fortney, J. J., Lecavelier des Etangs, A., \& Vidal-Madjar, A. 2012, MNRAS, 426, 1663

Sing, D. K., Pont, F., Aigrain, S., Charbonneau, D., Désert, J.-M., Gibson, N., Gilliland, R., Hayek, W., Henry, G., Knutson, H., Lecavelier Des Etangs, A., Mazeh, T., \& Shporer, A. 2011b, MNRAS, 416, 1443

Sing, D. K., Wakeford, H. R., Showman, A. P., Nikolov, N., Fortney, J. J., Burrows, A. S., Ballester, G. E., Deming, D., Aigrain, S., Désert, J.-M., Gibson, N. P., Henry, G. W. Knutson, H., Lecavelier des Etangs, A., Pont, F., Vidal-Madjar, A., Williamson, M. W., \& Wilson, P. A. 2015, MNRAS, 446 , 2428

Southworth, J. 2008, MNRAS, 386, 1644

Southworth, J., Bruntt, H., \& Buzasi, D. L. 2007, A\&A, 467, 1215

Tashkun, S. A., Perevalov, V. I., Teffo, J.-L., Bykov, A. D., \& Lavrentieva, N. N. 2003, Journal of Quantitative Spectroscopy and Radiative Transfer, 82, 165

Teske, J. K., Schuler, S. C., Cunha, K., Smith, V. V., \& Griffith, C. A. 2013, ApJ, 768, L12

Tinetti, G., Deroo, P., Swain, M. R., Griffith, C. A., Vasisht, G., Brown, L. R., Burke, C., \& McCullough, P. 2010, ApJ, 712, 